\title{
A retrospective study on the clinical characteristics and radiological features of primary pulmonary lymphoma
}

\author{
Xiaodong Xie, Lei Zhang, Mengjie Wu, Zheng Kang, Hongwei Yan, Xiuming Zhang, Wenrong Shen, \\ Min Dong
}

Departments of Radiology, Jiangsu Cancer Hospital, Jiangsu Institute of Cancer Research, Nanjing Medical University Affiliated Cancer Hospital, Nanjing 210000, China

Contributions: (I) Conception and design: W Shen, M Dong; (II) Administrative support: W Shen; (III) Provision of study materials or patients: X Xie, M Dong; (IV) Collection and assembly of data: X Xie, L Zhang, M Wu, Z Kang; (V) Data analysis and interpretation: X Xie, H Yan, X Zhang; (VI) Manuscript writing: All authors; (VII) Final approval of manuscript: All authors.

Correspondence to: Professor Wenrong Shen; Professor Min Dong. Department of Radiology, Jiangsu Cancer Hospital, Jiangsu Institute of Cancer Research, Nanjing Medical University Affiliated Cancer Hospital, 42 Baiziting, Nanjing 210000, China.

Email: jszlyyct@126.com; 13851670000@163.com.

Background: The retrospective study was mainly performed to determine the clinical symptoms and radiological characteristics of primary pulmonary lymphoma (PPL) to improve the recognition and diagnosis of the disease.

Methods: Between June 2007 and June 2019, the clinical data and radiological images of the 16 patients with PPL confirmed by pathology were retrospectively analyzed.

Results: Among the 16 patients with PPL (6 males and 10 females, aged 32 to 72 years, with a median age of 55.13 years), 9 patients were mucosa-associated lymphoid tissue lymphoma (MALT) and 7 patients were diffuse large B-cell lymphoma (DLBCL); all of the patients did not suffer from autoimmune disease [such as rheumatoid arthritis (RA), systemic lupus erythematosus (SLE), or Sjogren's syndrome (SSS)]; and 11 patients had a long-term smoking history from 10 to 40 years. The common clinical symptoms were as follows: chest discomfort $(n=8)$, cough $(n=10)$, chest pain $(n=7)$, fever $(n=6)$, apnea $(n=1)$, fatigue $(n=4)$ and weight loss $(n=3)$, however, 6 cases did not show clear symptoms at the time of diagnosis. Blood tests revealed anemia $(n=6)$, thrombocytopenia $(n=2)$, lactate dehydrogenase (LDH) level $(n=7)$, C-reactive protein $(C R P)$ $(n=9)$, erythrocyte sedimentation rate $(\mathrm{ESR})(\mathrm{n}=8)$ and no tumor-related indexes were detected abnormal. The chest radiological images showed a total of 8 cases with multiple masses, 2 cases with different types of nodes, 4 cases with patchy infiltration or consolidation shadow, with or without an air bronchogram, and 2 cases with a mixed manifestation. All the lesions were only involved in unilateral lung (13 right, 3 left), none of them located on bilateral lung fields. At the time of admission, the patients were misdiagnosed as lung cancer $(n=9)$, pneumonia $(n=5)$, tuberculosis $(n=1)$, and diffuse interstitial lung disease $(n=1)$. Then final pathological diagnosis was confirmed by surgery $(n=9)$, percutaneous lung biopsy $(n=5)$, and bronchoscopic biopsy $(\mathrm{n}=2)$.

Conclusions: PPL is a rare disease, though clinical symptoms and radiological characteristics are not typical, they serve as significant clues for the diagnosis and differential diagnosis. Accurate diagnosis mainly depends on histopathological examination, however, conducting a retrospectively study could improve and enrich our knowledge to the disease and reduce inappropriate treatments.

Keywords: Primary pulmonary lymphoma (PPL); radiological features; clinical characteristics; diagnosis

Submitted Nov 14, 2019. Accepted for publication Feb 04, 2020.

doi: $10.21037 /$ tcr.2020.02.16

View this article at: http://dx.doi.org/10.21037/tcr.2020.02.16 


\section{Introduction}

Lymphoma is a malignant tumor of lymphoid tissue and often affects the lungs through blood dissemination or directly invades from mediastinal/hilar lymph nodes, which rarely be detected as a form of primary pulmonary disease $(1,2)$. primary pulmonary lymphoma (PPL) is defined as clonal lymphoid proliferation affecting the lung, lobar, or primary bronchus, with or without mediastinal involvement, and absence of extra-thoracic lymphoma at the time of diagnosis or within the next 3 months (3-5). It is an uncommon tumor that accounts for only $0.4 \%$ of all malignant lymphomas and only $3-4 \%$ of extranodal lymphomas (6-8). Atypical clinical symptoms (such as chest cough, discomfort, fever, fatigue, etc.) and radiological characteristics of PPL lead to the difficulties in the disease diagnosis and treatment (9-11). The present knowledge of PPL is still limited and most studies on PPL are case reports, especially lacking of systematic clinical and radiological performances studies. In the current research, a total of 16 patients who attended our hospital and received operation or biopsy-proven PPL from June 2007 to June 2019 were systematically and comprehensively reviewed and analyzed. By analyzing and summarizing the clinical features, the laboratory examinations and radiological characteristics as well as radiological differential diagnosis of PPL, we aim to provide a better understanding to the disease, also improve the accuracy of early diagnosis and minimize the misdiagnosis.

\section{Methods}

\section{Patients}

The patients diagnosed as PPL were strictly according to the following criteria $(7,12)$ : (I) only unilateral or bilateral pulmonary was involved, with or without mediastinal involvement; (II) no previous extra-thoracic lymphoma was diagnosed; (III) no evidence of lymphoma involved in other organs upon diagnosis; (IV) no evidence of lymphoma was detected outside of the thorax within the 3 months following the initial diagnosis; $(\mathrm{V})$ disease confirmed by pathology. Here, totally 16 patients in our hospital were enrolled from June 2007 to June 2019.

\section{Clinical data}

Information on clinical findings, including age, gender, smoking history, past medical history, clinical symptoms, laboratory test results, radiological images, pathological results and follow-up data, were obtained from the clinical database and patient files. The data were systematically reviewed and analyzed.

\section{CT scanning protocol}

All the chest CT scans in our study were performed from the top of the thoracic cage to the level of lung base using Light Speed 64VCT (GE) and Discovery CT750 HD (GE). The scanning parameters were as follows: $120 \mathrm{kVp}, 110 \mathrm{~mA}$, slice thickness $5 \mathrm{~mm}$, matrix $512 \times 512$ and the acquisitions of images were in inspiration phases. High-resolution images were reconstructed at $1.25 \mathrm{~mm}$ slice thickness with a soft-tissue algorithm for mediastinal window setting (window width, $350 \mathrm{HU}$; window level, $50 \mathrm{HU}$ ), and a lung algorithm for lung window setting (window width, 1,500 HU; window level, -700 HU).

\section{Radiologic images analysis}

All the radiological images were evaluated and analyzed independently by two specialized chest radiologists. More attention was paid to the lesion size, location, shape, pleural thickness, pleural effusion, hilar or mediastinal lymphadenopathy. Definition of assessed CT features was referred to the previous study (13), and the 16 cases of PPL were categorized into different patterns based on the predominant radiological abnormalities. Decisions on CT scan findings were reached by consensus.

\section{Histologic evaluation}

Pathological diagnoses of PPL were based on histological examination of surgical specimens or bronchial, transbronchial or transthoracic biopsy material. The samples were obtained from all patients and reviewed by the pathologists.

\section{Statistical analysis}

Demographic and clinical data were presented as means, medians, minimum or maximum, frequencies and percentage. Continuous data between two different groups were compared using Student's $t$-test or Wilcoxon rank sum test, while categorical data were compared using $\chi^{2}$ test. The Statistical analysis was carried out with SPSS 20.0 version (Chicago, USA). $\mathrm{P}$ values less than 0.05 were considered 
Table 1 General clinical features in 16 patients with PPL

\begin{tabular}{|c|c|c|c|}
\hline Characteristics & MALT & DLBCL & Total No. \\
\hline Case No. & 9 & 7 & 16 \\
\hline \multicolumn{4}{|l|}{ Gender } \\
\hline Male & 4 & 2 & 6 \\
\hline Female & 5 & 5 & 10 \\
\hline Average age, years & 58.88 & 51.38 & $55.13[32-72]$ \\
\hline \multicolumn{4}{|l|}{ Smoking history } \\
\hline Yes & 5 & 6 & 11 \\
\hline No & 4 & 1 & 5 \\
\hline Autoimmune disease & 0 & 0 & 0 \\
\hline \multicolumn{4}{|l|}{ Common symptoms } \\
\hline Chest discomfort & 2 & 6 & 8 \\
\hline Cough & 3 & 7 & 10 \\
\hline Chest pain & 2 & 5 & 7 \\
\hline Fever & 1 & 5 & 6 \\
\hline Apnea & 0 & 1 & 1 \\
\hline Fatigue & 1 & 3 & 4 \\
\hline Weight loss & 1 & 2 & 3 \\
\hline No comfortable & 6 & 0 & 6 \\
\hline
\end{tabular}

PPL, primary pulmonary lymphoma; DLBCL, diffuse large B-cell lymphoma; MALT, mucosa-associated lymphoid tissue lymphoma.

statistically significant.

\section{Results}

\section{General clinical features}

As shown in Table 1, general clinical data of the $16 \mathrm{PPL}$ patients were listed. Among the 16 patients (6males and 10 females, aged 32 to 72 years, with a median age of 55.13 years), $9(56.25 \%)$ patients were mucosa-associated lymphoid tissue lymphoma (MALT) and 7 (43.75\%) patients were diffuse large B-cell lymphoma (DLBCL). The mean age in MALT group was 58.88 and in DLBCL groups was 51.38 . There were $11(68.75 \%)$ patients with a long-term smoking history range from 10 to 40 years. No one suffered from autoimmune disease (i.e., RA, SLE, SSS). The common clinical symptoms were as follows: chest discomfort $(\mathrm{n}=8 ; 50.00 \%)$, cough $(\mathrm{n}=10 ; 62.50 \%)$, chest pain $(n=7 ; 43.75 \%)$, fever $(n=6 ; 37.50 \%)$, apnea $(n=1 ; 6.25 \%)$,
Table 2 Blood tests in 16 patients with PPL

\begin{tabular}{lccc}
\hline Features & MALT & DLBCL & Total No. \\
\hline Case No. & 9 & 7 & 16 \\
Anemia & 2 & 4 & 6 \\
Thrombocytopenia & 0 & 2 & 2 \\
LDH & 1 & 6 & 7 \\
CRP & 4 & 5 & 9 \\
ESR & 2 & 6 & 8 \\
Tumor-related indexes & 0 & 0 & 0 \\
\hline
\end{tabular}

PPL, primary pulmonary lymphoma; DLBCL, diffuse large B-cell lymphoma; MALT, mucosa-associated lymphoid tissue lymphoma; LDH, lactate dehydrogenase; CRP, C-reactive protein; ESR, erythrocyte sedimentation rate.

fatigue $(\mathrm{n}=4 ; 25.00 \%)$ and weight loss $(\mathrm{n}=3 ; 18.75 \%)$. Noticeably, there were $6(66.67 \%)$ cases in MALT group with no symptoms at diagnosis. Table 1 may also reveal that patients in DLBCL group suffered relative severer clinical symptoms compared with those in MALT group.

\section{Abnormal blood indexes}

The results of blood tests were shown in Table 2 . The abnormal indexes were anemia $(n=6 ; 37.50 \%)$, thrombocytopenia $(\mathrm{n}=2 ; 12.50 \%)$, lactate dehydrogenase (LDH) level ( $\mathrm{n}=7 ; 43.75 \%)$, C-reactive protein (CRP) level $(\mathrm{n}=9 ; 56.25 \%)$ and erythrocyte sedimentation rate (ESR) level $(\mathrm{n}=8 ; 50.00 \%)$. Here, the tumor-related indexes of the 16 patients were normal. In the two groups, we found that the abnormal rate of blood indexes in MALT group was lower than that in DLBCL group. In MALT group, the highest rate of abnormal index was CRP (44.44\%, $n=4 / 9)$, while in DLBCL group, the abnormal percentages were $57.14 \%(\mathrm{n}=4 / 7), 28.57 \%(\mathrm{n}=2 / 7), 85.71 \%(\mathrm{n}=6 / 7), 71.43 \%$ $(\mathrm{n}=5 / 7), 85.71 \%(\mathrm{n}=6 / 7)$ in anemia, thrombocytopenia, LDH, CPR and ESR, respectively.

\section{Radiological images features}

Based on the predominant radiological features of the 16 patients, the lesions were classified into mass-type, nodetype, patch-like or consolidate type and mixed-type. As shown in Table 3, 8 cases showed multiple masses, 2 cases showed different (single or more) nodes, 4 cases had patchlike infiltration or consolidation shadow with or without air 
Table 3 CT manifestations in 16 patients with PPL

\begin{tabular}{lccc}
\hline Characteristic & MALT & DLBCL & Total No. \\
\hline Case No. & 9 & 7 & 16 \\
Pattern & 2 & 6 & 8 \\
Mass-type & 2 & 0 & 2 \\
Node-type & 4 & 0 & 4 \\
Patch or & 1 & 1 & 2 \\
consolidate type & & & \\
Mixed-type & 9 (8 right; 1 left) & 7 (5 right; 2 left) & 16 \\
Location & 0 & 0 & 0 \\
Unilateral & 1 & 3 & 4 \\
Bilateral & 0 & 2 & 2 \\
Pleural thickness & 1 & 3 & 4 \\
Pleural effusion & & & \\
Hilar or mediastinal & lymphadenopathy & &
\end{tabular}

PPL, primary pulmonary lymphoma; DLBCL, diffuse large B-cell lymphoma; MALT, mucosa-associated lymphoid tissue lymphoma.

bronchogram, and 2 cases showed a mixed manifestation. In DLBCL group, 6 out of 7 patients presented the mass-type, and only 1 case showed mixed-type. While in MALT group, the 4 different types all could be detected. The distribution of the lesions was in unilateral lung $(100 \%, n=16 / 16)$, especially in the right lung ( $\mathrm{n}=13 / 16)$, and no case involved a bilateral lung. The presentations of pleural thickness, pleural effusion and hilar or mediastinal lymphadenopathy were relatively easier to be detected in the DLBCL cases, and the incidence of the three manifestations were $42.86 \%$ $(\mathrm{n}=3 / 7), 28.57 \%(\mathrm{n}=2 / 7), 42.86 \%(\mathrm{n}=3 / 7)$, respectively, whereas they were only $11.11 \%(n=1 / 9), 0 \%(n=0 / 9)$, $11.11 \%,(n=1 / 9)$ in the MALT cases, respectively.

\section{Mass-type}

A total of 8 patients were mass-type (2 MALT, 6 DLBCL). The size of the mass ranged from $3.43 \mathrm{~cm} \times 2.80 \mathrm{~cm}$ to $10.90 \mathrm{~cm} \times 4.40 \mathrm{~cm}$, with irregular morphology and uneven density. Local necrosis could be observed in some larger mass, and small calcification lesions in the marginal zone were also detected. When underwent a contrastenhanced CT scan, the lesion showed a trend of gradual intensification. Of the 8 patients, 2 presented multiple lesions (Figure 1), and 6 showed a single lesion on the right lobe. Pleural effusion, thickening or involvement of the pleura, and enlargement of the hilar or mediastinal lymph nodes were relatively easy to be detected in the Mass type.

\section{Node-type}

Two cases presented single node type. The nodes were slightly irregular in shape and slightly inhomogeneous in density. In one case, burrs and lobulated presence could be seen at the margin and the sign of air bronchogram was also detected (Figure 2). In another case, nodules and parabronchial lymph nodes were observed. The lesion in the inferior lobe of right lung proved to be an MALT exactly, and the adenopathy was invaded by the MALT lesion (Figure 3).

\section{Patch or consolidate type}

All the 4 patients were MALT-type, characterized by flaky or patchy dense shadow with the showing signs of air bronchogram, or pulmonary infiltrate with diffuse margins (Figure 4).

\section{Mixed-type}

Two patients were mixed-type (1 MALT, 1 DLBCL). Two or more manifestations of nodes, masses, consolidate shadow or interstitial/crazy paving pattern could be seen simultaneously in one patient (Figure 5).

\section{Diagnostic results and methods}

As the clinical symptoms and radiological features are atypical, the disease is difficult to be diagnosed. As shown in Table 4, the patients were misdiagnosed as lung cancer $(\mathrm{n}=9 / 16,56.25 \%)$, pneumonia $(\mathrm{n}=5 / 16,31.25 \%)$, tuberculosis $(\mathrm{n}=1 / 16,6.25 \%)$ and diffuse interstitial lung disease $(n=1 / 16,6.25 \%)$. The results were finally confirmed by surgery $[\mathrm{n}=9,2$ by open lung biopsy and 7 by videoassisted thoracic surgery (VATS)], percutaneous lung biopsy $(\mathrm{n}=5)$, and bronchoscopic biopsy $(\mathrm{n}=2)$.

\section{Differential diagnosis}

Before confirmation, our cases were misdiagnosed as pneumonia, lung cancer, or tuberculosis. Discussion on differential diagnosis between PPL and the mentioned diseases were necessary:

\section{For patch-like or consolidate type PPL}

This type of PPL often accompanied with sign of air 

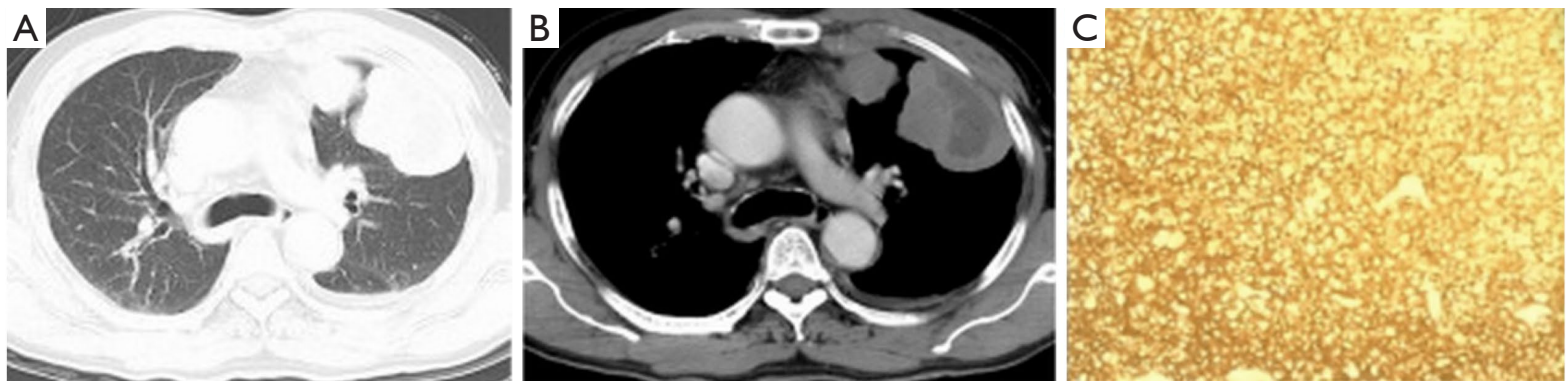

Figure 1 Mass-type: two lesions occupied the upper left lung, with irregular shape and lobulated presence, local necrosis detected in the larger mass. Both lesions were close to the chest wall without distinct boundaries (A,B). A small amount of pleural effusion can be detected (B). Proved to be a DLBCL by immunohistochemistry $(\times 100, C)$. DLBCL, diffuse large B-cell lymphoma.
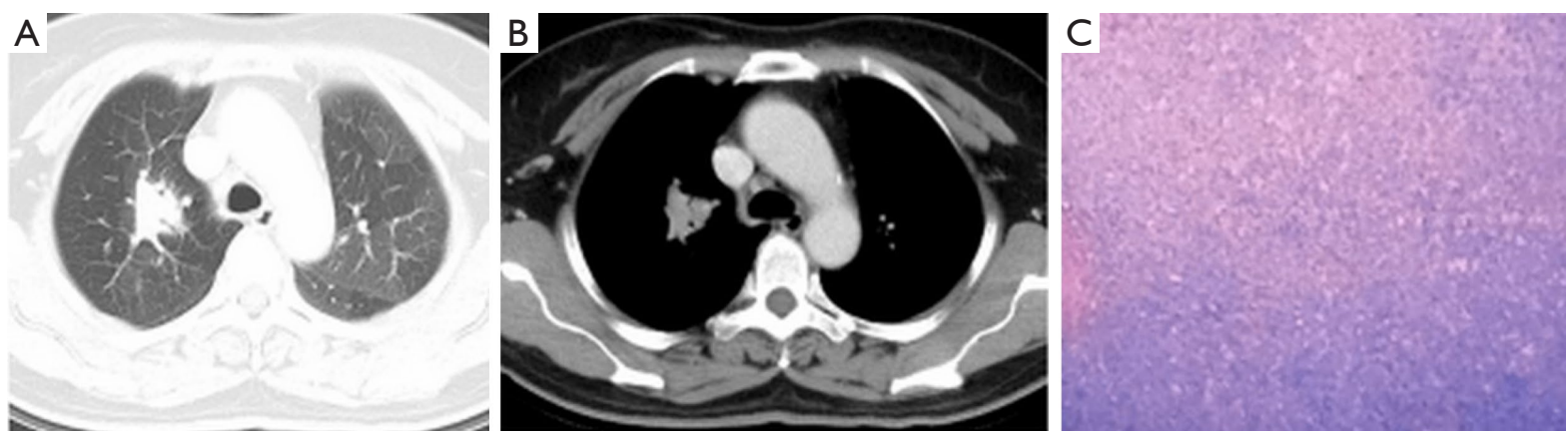

Figure 2 Node type: an irregular dense nodule was detected in the upper right lobe, and burrs and lobulated presence could be observed $(\mathrm{A}, \mathrm{B})$. The air bronchogram sign could be found in this case (A). Proved to be a MALT by HE staining method ( $\times 100$, C). MALT, mucosaassociated lymphoid tissue lymphoma.
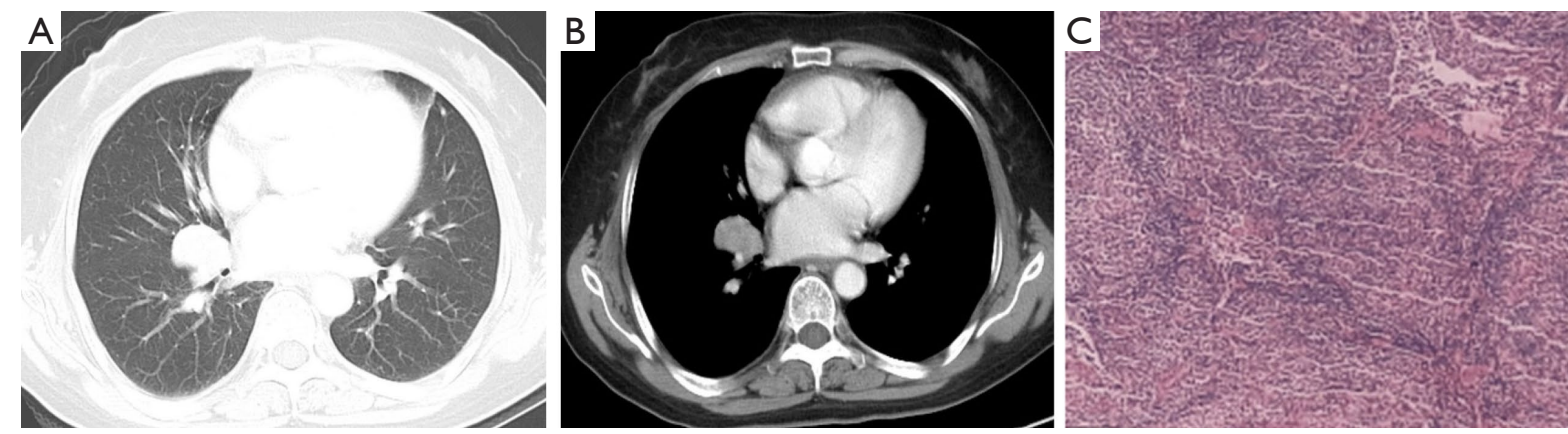

Figure 3 Node type: a nodule located at the perihilar region of right lung and could not be isolated with the peribronchial lymph node(A,B). Also, subcarinal lymph node enlargement was observed (B). Proved to be a MALT by HE staining method ( $\times 100$, C). MALT, mucosaassociated lymphoid tissue lymphoma.

bronchogram, and bronchiectatic changes in our study and previously reported $(6,9,14)$. Additionally, antiinflammatory treatment is ineffective to PPL. This type of PPL should be distinguished from (I) lobar pneumonia, which is characterized by consolidation of pulmonary segments or lobes with normal internal bronchi, moreover, patients may benefit from anti-inflammatory treatment; and from (II) pneumonia type carcinoma of lung (PTCL), the air bronchogram sign could be observed, whereas the bronchial wall is often irregular, narrow, interrupted or 

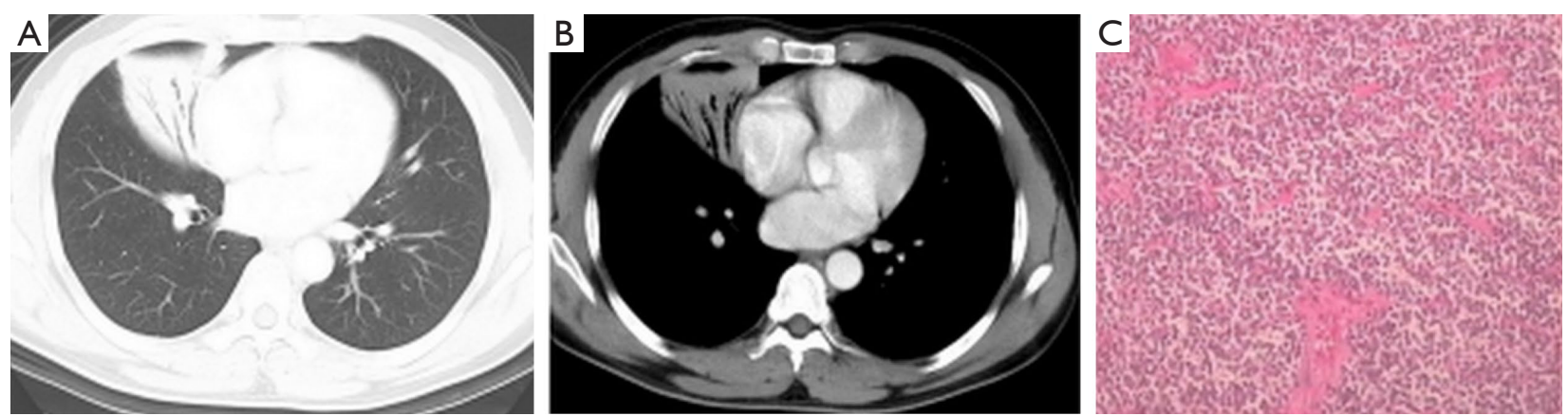

Figure 4 Patch or consolidate type: the dense shadow in the middle lobe of the right lung with the sign of air bronchogram (A,B). Proved to be a MALT by HE staining method $(\times 100, C)$. MALT, mucosa-associated lymphoid tissue lymphoma.
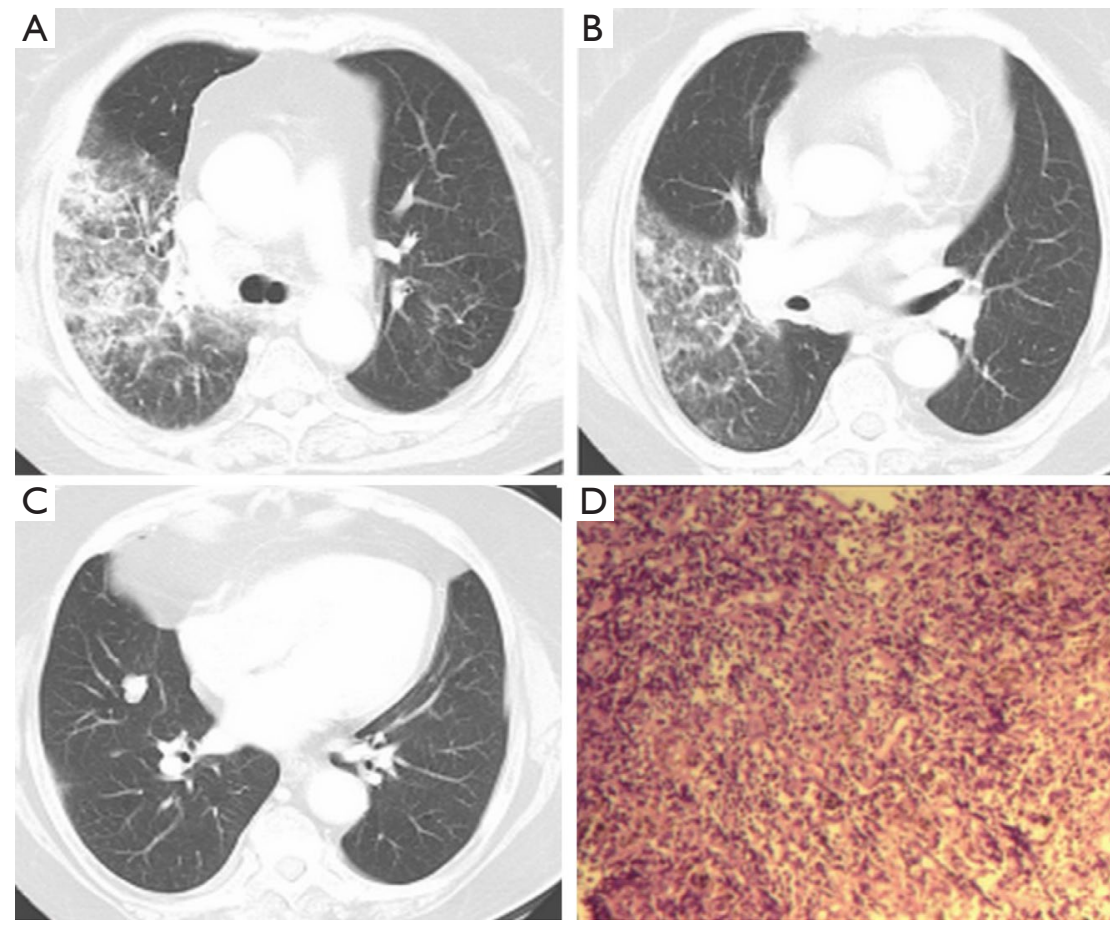

Figure 5 PMixed-type: an interstitial/crazy paving pattern as well as two small nodules were observed (A,B,C). Proved to be a MALT by immunohistochemistry $(\times 100, \mathrm{D})$. MALT, mucosa-associated lymphoid tissue lymphoma.

thickened.

For node or mass-type PPL: should be separated from: (I) Peripheral lung cancer, which shows a mass or node with lobulated shape, spicules of margin, pleural indentation, vessel convergence and vacuole signs. However, the signs were less observed in node or mass-type PPL, especially the pleural indentation, vessel convergence and vacuole signs in our patients. Additionally, for peripheral lung cancer, the tumors apparently strengthened after a contrastenhanced scan and often accompanied with other malignant signs, such as lymph node enlargement, pleural effusion, or distant metastasis.

(II) Tuberculoma. Tuberculoma predilection sites were in upper and lower dorsal segments and often surrounded b y several smaller satellite lesions. The lesion with calcificati on or peripheral calcification would offer important clues in differential diagnosis. Also, anti-tuberculosis treatment was 
Table 4 Diagnostic results and methods

\begin{tabular}{lccc}
\hline Characteristic & MALT & DLBCL & Total No. \\
\hline Case No. & 9 & 7 & 16 \\
Misdiagnosis & 2 & 7 & 9 \\
Lung cancer & 5 & 0 & 5 \\
Pneumonia & 1 & 0 & 1 \\
Tuberculosis & 1 & 0 & 1 \\
$\begin{array}{l}\text { Diffuse interstitial lung } \\
\text { disease }\end{array}$ & & & \\
Confirmed by & 0 & 2 & 2 \\
Open lung & 5 & 2 & 7 \\
Vast & 1 & 4 & 5 \\
Percutaneous lung biopsy & 1 & 1 & 2 \\
Bronchoscopic biopsy & & & \\
\hline
\end{tabular}

DLBCL, diffuse large B-cell lymphoma; MALT, mucosaassociated lymphoid tissue lymphoma.

effective to patients with tuberculoma.

(III) Metastatic tumor. These patients usually had a history of malignant tumor.

\section{Discussion}

PPL is a rare disease, accounting for only $0.4 \%$ of all lymphomas cases $(5,12)$, and such a rarity is less considered in diagnosis of lung lesions, thus leading to inappropriate treatments. The most common type of PPL is MALT lymphoma, and the DLBCL is less frequent $(12,15-17)$. In the current study, MALT $(n=9)$ and DLBCL $(n=7)$ were all observed in the 16 patients. By carefully analyzing and summarizing the clinical features, the laboratory examinations and radiological characteristics as well as radiological differential diagnosis of PPL, we could gain a better understanding to this disease and minimize the inappropriate treatments.

PPL has various clinical symptoms, including cough, chest pain, fever, apnea, fatigue, weight loss, night sweats, etc. These non-specific symptoms and physical signs do not contribute significantly to PPL diagnosis, and noticeably, at least one-third of the patients have no symptoms $(6,18)$. In our study, $62.50 \%(n=10 / 16)$ patients presented some different common clinical symptoms, while $37.50 \%$ $(n=6 / 16)$ showed no symptoms, which was consisted with previous reports $(4-6,8,18)$.Here the 6 ones without symptoms were MALT patients, and the DLBCL patients presented relatively severer clinical symptoms than that of MALT.

In this study, the number of male and female patients is 6 and 10, respectively, showing a female preponderance and is inconsistent with the previous reports $(19,20)$. The age of our patients ranges from 32 to 72 years, with an average age of 55.13 years. The mean age in MALT group was 58.88 and in DLBCL groups was 51.38. The age at PPL incidence in our study was younger than that in the previous reports, in which patients at their sixties and seventies have the highest incidence $(6,20,21)$. We inferred the reason could be attributed to the patients' increased health awareness and participation in physical examination. Most investigators considered that PPL was acquired in response to long-term exposure to various antigenic stimuli, such as smoking, infection, or autoimmune disease $(15,22-25)$. In our study, $68.75 \%(\mathrm{n}=11 / 16)$ patients have a long history of smoking, supporting that smoking could be an inducefactor to PPL. However, patients with autoimmune diseases are not identified in this study. In blood tests, patients were often found abnormal in anemia, thrombocytopenia, and elevated LDH levels (20). Additionally, we also observed the elevated CPR levels and accelerated ESR, but patients did not show abnormalities in tumor-related indicators. In the DLBCL group, the abnormal rate of blood parameters in patients is higher than that in the MALT group, and the clinical symptoms are relatively obvious, which may predict the poorer prognosis than that of MALT lymphoma (26). The similarities and differences in clinical features of PPL between our study and other studies indicated that this disease is quite complex and hard to be thoroughly clarified.

To our knowledge, CT is a very convenient and sensitive method for diagnosing lung lesions. However, PPL manifests itself in many different ways (21,27-31), which makes it difficult to be distinguished from lung cancer, pneumonia, tuberculosis, diffuse interstitial lung disease, in our study, especially from lung cancer. In the DLBCL group, 6 out of the 7 cases are mass type, and they were misdiagnosed as lung cancer until pathologically confirmed. We inferred this may be due to the facts that: on one hand the mass itself with similar characteristics to the lung cancer and make it hard to be distinguished from, on the other hand patients attending our cancer hospital tend to suffer from cancers, especially lung cancer in this area. In the MALT group, patch-like or consolidation types are relatively common, which is consistent with previous studies (9). And we have a high risk of misdiagnosing them 
as inflammatory lesions. Nowadays, PET-CT shows some advantages in proper diagnosis, staging and management in PPL $(32,33)$, more creative and detailed work could to be done to help minimize the misdiagnosis.

In conclusion, by retrospective analysis of the 16 cases, we have acquired a better understanding on PPL and would share some tips with everyone. In radiographic images, lesions of PPL could show as mass, node, patch, consolidate and mixed types, etc. Patch or consolidate type with or without air bronchogram was more common in MALT, while the mass type was more inclined to DLBCL. The presentations of pleural thickness, pleural effusion and hilar or mediastinal lymphadenopathy were relatively easier to be detected in the DLBCL. Although the clinical symptoms and blood indexes were not typical, we also found that the DLBCL patients suffered relatively severer clinical symptoms than that of MALT. And the level of LDH, CRP, and ESR indexes showed elevated, especially in DLBCL. When patients over 50 years and radiographic images showed as we reported before, in particular for those with ineffective anti-infection and long course of disease that have poor therapeutic results, PPL should be considered. Once PPL is suspected, a differential diagnosis should be done with pneumonia, lung cancer, tuberculosis, etc., as we mentioned above and an early biopsy or puncture is recommended to avoid inappropriate treatments.

There are also several limitations in this study. First, this is a retrospective study and the sample size is small (due to the rarity of this disease), which would weaken the accuracy and reliability of the results. Second, the heterogeneous features of our Sample (for example some patients only underwent plain CT scan, lacking contrast-enhanced CT information).

\section{Acknowledgments}

Funding: The present study was supported by the Jiangsu Cancer Hospital College Project (Jiangsu, China; grant No. ZN201611).

\section{Footnote}

Conflicts of Interest: All authors have completed the ICMJE uniform disclosure form (available at http://dx.doi. org/10.21037/tcr.2020.02.16). The authors have no conflicts of interest to declare.

Ethical Statement: The authors are accountable for all aspects of the work in ensuring that questions related to the accuracy or integrity of any part of the work are appropriately investigated and resolved. The study was conducted in accordance with the Declaration of Helsinki (as revised in 2013). Ethical approval for this investigation was obtained from the Ethics Committee of Jiangsu Cancer Hospital (Nanjing, Jiangsu, China) (No. ZN201611). And all patients consent to participate and all data were consent for publish.

Open Access Statement: This is an Open Access article distributed in accordance with the Creative Commons Attribution-NonCommercial-NoDerivs 4.0 International License (CC BY-NC-ND 4.0), which permits the noncommercial replication and distribution of the article with the strict proviso that no changes or edits are made and the original work is properly cited (including links to both the formal publication through the relevant DOI and the license). See: https://creativecommons.org/licenses/by-nc-nd/4.0/.

\section{References}

1. Miller DL, Allen MS. Rare pulmonary neoplasms. Mayo Clin Proc 1993;68:492-8.

2. Freeman C, Berg JW, Cutler SJ. Occurrence and prognosis of extranodal lymphomas. Cancer 1972;29:252-60.

3. Saltzstein SL. Pulmonary malignant lymphomas and pseudolymphomas: Classification, therapy, and prognosis. Cancer 1963;16:928-55.

4. Tang VK, Vijhani P, Cherian SV, et al. Primary pulmonary lymphoproliferative neoplasms. Lung India 2018;35:220-30.

5. Tanveer S, Damati AE, Baz AE, et al. Primary Pulmonary Hodgkin Lymphoma. Rare Tumors 2015;7:5968.

6. Parissis H. Forty years literature review of primary lung lymphoma. J Cardiothorac Surg 2011;6:23.

7. Wróbel T, Dzietczenia J, Prochorec-Sobieszek M, et al. Primary pulmonary diffuse large B-cell lymphoma. Am J Hematol 2012;87:107-8.

8. Graham BB, Mathisen DJ, Mark EJ, et al. Primary pulmonary lymphoma. Ann Thorac Surg 2005;80:1248-53.

9. Yao D, Zhang L, Wu PL, et al. Clinical and misdiagnosed analysis of primary pulmonary lymphoma: a retrospective study. BMC Cancer 2018;18:281.

10. Yoshino N, Hirata T, Takeuchi C, et al. Primary Pulmonary Mucosa-Associated Lymphoid Tissue Lymphoma with a Nodular Opacity: Report of a Case. J Nippon Med Sch 2017;84:87-9. 
11. Yang X, Xu X, Song B, et al. Misdiagnosis of primary pleural DLBCL as tuberculosis: A case report and literature review. Mol Clin Oncol 2018;8:729-32.

12. Cooksley N, Judge DJ, Brown J. Primary pulmonary Hodgkin's lymphoma and a review of the literature since 2006. BMJ Case Rep 2014. doi: 10.1136/bcr-2014-204020.

13. Austin JH, Müller NL, Friedman PJ, et al. Glossary of terms for CT of the lungs: recommendations of the Nomenclature Committee of the Fleischner Society. Radiology 1996;200:327-31.

14. Lee J, Park H, Kim YW, et al. Pulmonary Lymphoma Misdiagnosed as Pneumonia. Indian J Hematol Blood Transfus 2016;32:509-11.

15. Kurtin PJ, Myers JL, Adlakha H, et al. Pathologic and clinical features of primary pulmonary extranodal marginal zone B-cell lymphoma of MALT type. Am J Surg Pathol 2001;25:997-1008.

16. Li G, Hansmann ML, Zwingers T, et al. Primary lymphomas of the lung: morphological, immunohistochemical and clinical features. Histopathology 1990;16:519-31.

17. Schulz R, Baseler G, Ghofrani HA, et al. Nocturnal periodic breathing in primary pulmonary hypertension. Eur Respir J 2002;19:658-63.

18. Cordier JF, Chailleux E, Lauque D, et al. Primary pulmonary lymphomas: A clinical study of 70 cases in nonimmunocompromised patients. Chest 1993;103:201-8.

19. Tanriverdi E, Acat M, Ozgul G, et al. Primary pulmonary lymphoma: four different and unusual radiologic and clinical manifestations. Leuk Lymphoma 2017;58:1231-3.

20. Hu YH, Hsiao LT, Yang CF, et al. Prognostic factors of Chinese patients with primary pulmonary non-Hodgkin's lymphoma: the single-institute experience in Taiwan. Ann Hematol 2009;88:839-46.

21. Majid N, Kamal EB, Oncology B, et al. Primary pulmonary lymphoma: About five cases and literature review. Lung India 2014;31:53-5.

22. Imai H, Sunaga N, Kaira K, et al. Clinicopathological Features of Patients with Bronchial-Associated Lymphoid Tissue Lymphoma. Intern Med 2009;48:301-6.

Cite this article as: Xie X, Zhang L, Wu M, Kang Z, Yan H, Zhang X, Shen W, Dong M. A retrospective study on the clinical characteristics and radiological features of primary pulmonary lymphoma. Transl Cancer Res 2020;9(3):1969-1977. doi: $10.21037 /$ tcr.2020.02.16
23. Borie R, Wislez M, Thabut G, et al. Clinical characteristics and prognostic factors of pulmonary MALT lymphoma. Eur Respir J 2009;34:1408-16.

24. Ahmed S, Kussick SJ, Siddiqui AK, et al. Bronchialassociated lymphoid tissue lymphoma: a clinical study of a rare disease. Eur J Cancer 2004;40:1320-26.

25. Yu H, Chen G, Zhang R, et al. Primary Intravascular large B-cell lymphoma of lung: a report of one case and review. Diagn Pathol 2012;7:70.

26. Zinzani PL, Martelli M, Poletti V, et al. Practice guidelines for the management of extranodal non-Hodgkin's lymphomas of adult non-immunodeficient patients. Part I: primary lung and mediastinal lymphomas. A project of the Italian Society of Hematology, the Italian Society of Experimental Hematolo. Haematologica 2008;93:1364-71.

27. Bae YA, Lee KS, Han J, et al. Marginal zone B-cell lymphoma of bronchus-associated lymphoid tissue: imaging findings in 21 patients. Chest 2008;133:433-40.

28. Li H, Wang T, Wei X, et al. Marginal zone B?cell lymphoma of the pulmonary mucosa? associated lymphoid tissue: A case report. Oncol Lett 2015;10:1731-34.

29. Cozzi D, Dini C, Mungai F, et al. Primary pulmonary lymphoma: imaging findings in 30 cases. Radiol Med 2019;124:1262-9.

30. Chen Y, Chen AP, Jiang HL, et al. HRCT in primary pulmonary lymphoma: can CT imaging phenotypes differentiate histological subtypes between mucosaassociated lymphoid tissue (MALT) lymphoma and nonMALT lymphoma? J Thorac Dis 2018;10:6040-9.

31. Furuya K, Yasumori K, Takeo S, et al. Lung CT: Part 1, Mimickers of lung cancer--spectrum of CT findings with pathologic correlation. AJR Am J Roentgenol 2012;199:W454-63.

32. Xu H, Xu K, Wang R, et al. Primary Pulmonary Diffuse Large B-Cell Lymphoma on FDG PET/CT-MRI and DWI. Medicine 2015;94:e1210.

33. Albano D, Borghesi A, Bosio G, et al. Pulmonary mucosa-associated lymphoid tissue lymphoma: $18 \mathrm{~F}-\mathrm{FDG}$ PET/CT and CT findings in 28 patients. Br J Radiol 2017;90:20170311. 